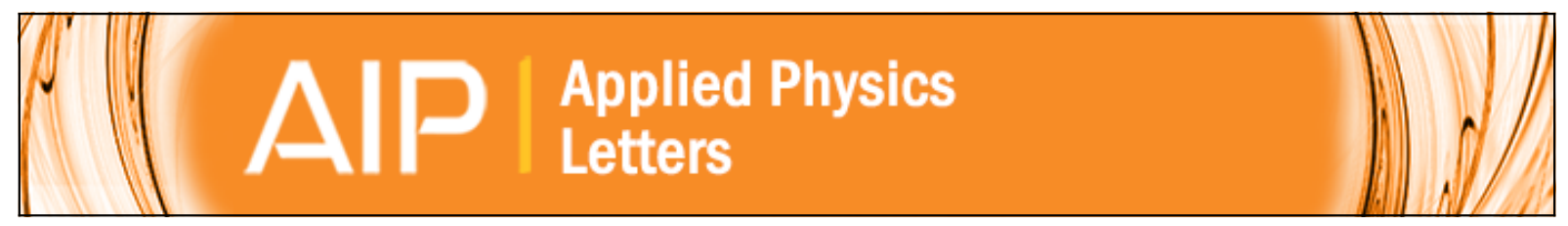

\title{
Fiber Bragg grating vacuum sensors
}

Ben McMillen, Chuck Jewart, Michael Buric, Kevin P. Chen, Yuankun Lin, and Wei Xu

Citation: Applied Physics Letters 87, 234101 (2005); doi: 10.1063/1.2140082

View online: http://dx.doi.org/10.1063/1.2140082

View Table of Contents: http://scitation.aip.org/content/aip/journal/apl/87/23?ver=pdfcov

Published by the AIP Publishing

\section{Articles you may be interested in}

Measurement error of surface-mounted fiber Bragg grating temperature sensor

Rev. Sci. Instrum. 85, 064905 (2014); 10.1063/1.4885463

A multiplexed fiber Bragg grating sensor for simultaneous salinity and temperature measurement J. Appl. Phys. 103, 053107 (2008); 10.1063/1.2890156

Cryogenic Fiber Optic Temperature Sensors Based on Fiber Bragg Gratings AIP Conf. Proc. 823, 267 (2006); 10.1063/1.2202425

Dual temperature and strain sensor using a combined fiber Bragg grating and fluorescence intensity ratio technique in $\mathrm{Er} 3+$-doped fiber

Rev. Sci. Instrum. 74, 2880 (2003); 10.1063/1.1569406

Fiber optic sensor for dual measurement of temperature and strain using a combined fluorescence lifetime decay and fiber Bragg grating technique

Rev. Sci. Instrum. 72, 3186 (2001); 10.1063/1.1372171

\section{A|P| $\left.\right|_{\text {Applied Physics }} ^{\text {Journal of }}$}

Journal of Applied Physics is pleased to announce André Anders as its new Editor-in-Chief 


\title{
Fiber Bragg grating vacuum sensors
}

\author{
Ben McMillen, Chuck Jewart, Michael Buric, and Kevin P. Chen ${ }^{a}$ \\ Department of Electrical and Computer Engineering, University of Pittsburgh, Pittsburgh, Pennsylvania \\ 15261 \\ Yuankun Lin \\ Department of Physics and Geology, University of Texas-Pan American, Edinburg, Texas 78541
}

Wei Xu

StockerYale, Inc., Salem, New Hamphire 03079

(Received 16 May 2005; accepted 24 October 2005; published online 28 November 2005)

\begin{abstract}
This letter demonstrates functional enhancements of fiber Bragg grating sensors powered by in-fiber light. High-power laser light transmitted in double-clad optical fiber was extracted from the fiber core to heat an on-fiber metal coating. When the power-laser is turned off, the fiber Bragg grating is used as a passive component for temperature sensing. When the laser is turned on, the thermal response of the optically heated grating was used to monitor ambient air pressure. The sensitivity and dynamic range of optically powered fiber sensors can be actively adjusted by in-fiber light to measure vacuum pressures over four orders of magnitude. (C) 2005 American Institute of Physics.

[DOI: $10.1063 / 1.2140082$ ]
\end{abstract}

Since their discovery in 1978, fiber Bragg grating $^{1}$ (FBG) sensors have been widely used for a number of sensing applications including temperature, stress, pressure, and refractive index change measurements. ${ }^{2,3}$ FBG sensors enjoy a number of advantages including long lifetime, immunity to electromagnetic noise, and ease of mulitiplexing. However, the applications and functionality of FBG sensors are limited by their total passivity. Passive sensors can only gather limited information. Once deployed; set point, sensitivity, trigging time, responsivity, and dynamic range for each individual fiber sensor cannot be adjusted or reset to adapt to the changing environment for active sensing. To enhance the functionality of a passive fiber sensor, we have proposed an active sensing concept by which an FBG sensor can be directly powered and controlled by in-fiber light carried in the same fiber. ${ }^{4}$ In this letter, we demonstrate the functional enhancement of a dual-function active FBG sensor for simultaneous vacuum and temperature measurement.

Thermal-based vacuum sensors such as Pirani gauges are among the most widely used instruments for vacuum measurement. ${ }^{5-7}$ In the past several years significant progress has been made toward the miniaturization of vacuum sensors using micro-electromechanical systems (MEMS) technology. Miniaturized vacuum sensors provide some important advantages including low fabrication cost, low power consumption, higher measurement sensitivity, and improved dynamic range. However, the implementation of miniaturized gauges in vacuum systems still requires a number of electrically isolated feed-throughs and a flange with a nominal width of 1 to 2 in. In situations where pressure information is needed at multiple points, such as in a spacecraft, a large number of electric wires, feed-throughs, and vacuum flanges will increase the complexity, risk of leaks, and cost of the measurement system. Moreover, long electrical cables are often needed to transmit perturbation-sensitive signals to a remotely located controller, which makes the measurement vulnerable to electromagnetic interference.
In this letter, we present an all-fiber thermal-based pressure gauge using fiber Bragg gratings (FBGs) without the need of a single electric cable. The fiber vacuum sensor demonstrated in this paper is based on the concept of active fiber components powered by in fiber laser light. ${ }^{4}$ Optical power transmitted in optical fiber was extracted from the fiber core to heat an on-fiber metal coating. The thermal response of the optically heated FBG was used to monitor the thermal response of the surrounding vacuum. Using wavelength division multiplexing (WDM) or time division multiplexing (TDM) technology, a large number of vacuum sensors can be inscribed in a single fiber with only one fiber feedthrough and one flange to provide pressure information at multiple points with minimal wiring. The one-fiber vacuum sensor presented in this paper promises a simple, light weight, agile, multi-functional vacuum and temperature sensing solution that is free from electromagnetic interference.

The FBG vacuum sensor presented here was inscribed in photosensitive double-clad fiber shown in Fig. 1. This fiber has a germanium and phosphorous co-doped silica core. The germanium concentration is 11 mole $\%$ and has a cut-off wavelength of $1450 \mathrm{~nm}$. The fiber was dual coated with UV curable acrylate with a diameter of $245 \mu \mathrm{m}$. The primary coating has the refractive index lower than that of pure silica and serves as the second cladding with 0.48 NA. The back-

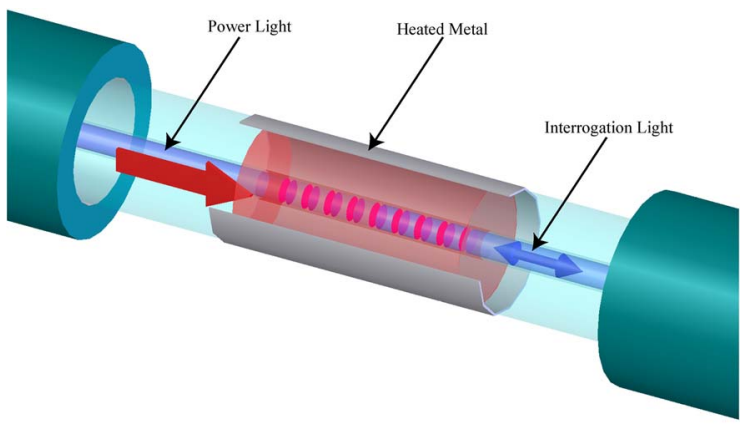

FIG. 1. (Color online) Schematic of active FBG vacuum sensor in a doubleclad fiber.

${ }^{a)}$ Electronic mail: kchen@engr.pitt.edu 


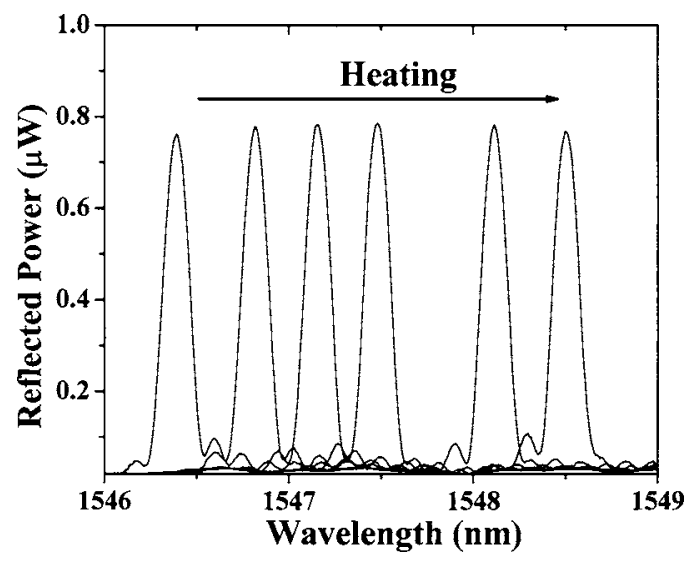

FIG. 2. Resonance wavelength shift of a $5 \mathrm{~mm}$ optically heated FBG at 173 mTorr. The optical power used to heat the FBG is $0,10.5,31,52,104$, and $145 \mathrm{~mW}$, respectively.

ground loss of the coating is $2.3 \mathrm{~dB} / \mathrm{km}$ around $1100 \mathrm{~nm}$. The inner cladding of the fiber was used to deliver highpower laser light to any point along the fiber for on-fiber optical heating. To inscribe an FBG in the fiber core, four-cm of the fiber coating was carefully removed using methylenechloride. A $5 \mathrm{~mm}$ long apodized uniform FBG was inscribed with a $\mathrm{KrF} 248 \mathrm{~nm}$ excimer laser using a standard phase mask technique. A silver coating ( $2 \mathrm{~cm}$ long) was then plated on the center of the stripped fiber where the FBG is located. The silver coating is estimated to be $0.2 \mu \mathrm{m}$ thick. The double-clad fiber was then inserted into a vacuum calibration chamber through two fiber feed-throughs. The pressure of the chamber was controlled by a rotary-vane pump and a mass flow controller connected to the ambient atmosphere. A convection gauge installed next to the FBG was used to measure and calibrate the chamber pressure. The reflection spectra of the FBG were monitored with an optical spectrum analyzer (Ando 6317C) and a broadband source via a circulator connected to the other end of the fiber.

High-power multiwavelength Ar-ion laser light (0.1-4 $\mathrm{W})$ is coupled into the inner cladding of the fiber from one end using a $20 \times$ microscope objective. The coupling efficiency was over $90 \%$. When the argon laser is turned off, the FBG is used as a passive sensor to gauge precisely the ambient temperature of the vacuum chamber. When the argon laser is turned on, a portion of argon laser light propagating through the uncoated section of fiber is absorbed by the silver coating. It was estimated that approximately $40 \%$ of the input power was absorbed by the $2 \mathrm{~cm}$ long metal coating. The total absorption of the power light depends on the coating properties including length, and geometry of the metal. We found that the fiber temperature distribution and birefringence can be thermally tailored by selectively removing the fiber coating along either the longitudinal direction or the azimuth direction of the fiber. The laser light absorbed by the uniform silver coating produces a uniform temperature profile, which leads to a resonance wavelength shift without distorting the reflection spectrum. Figure 2 shows the resonance wavelength shift of a $5 \mathrm{~mm}$ FBG at 173 mTorr. It was observed that $145 \mathrm{~mW}$ input power from the argon ion laser uniformly shifts the resonance wavelength of the FBG to 2.0 $\mathrm{nm}$. Although the silver film presents a much larger thermal expansion coefficient of $5.5 \times 10^{-6} \mathrm{~K}$ than that of silica glass of $0.66 \times 10^{-6} \mathrm{~K}$, the very thin silver film $(<1 \mu \mathrm{m})$ does not alter the thermal response of the fiber significantly. There-

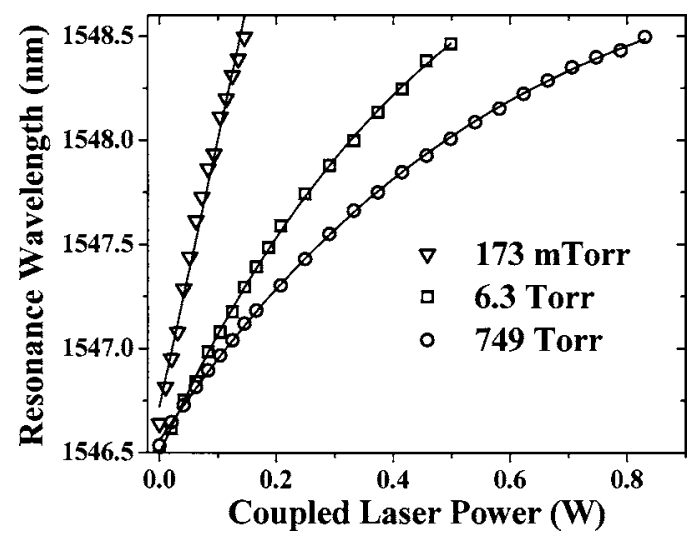

FIG. 3. Resonance wavelength shift of a $5 \mathrm{~mm}$ FBG as a function of coupled laser power at 173 mTorr, 6.3 Torr, and 745 Torr, respectively.

fore, the temperature rise of the FBG is estimated as $192{ }^{\circ} \mathrm{C}$ $\left(13 \mathrm{pm} /{ }^{\circ} \mathrm{C}^{2}\right)$. This temperature is similar to the operational temperature of Pirani gauge or a miniaturized MEMS vacuum sensor. Figure 3 shows the resonance wavelength shift of the FBG as a function of coupled argon ion laser power. The thermal exchange between the silver molecules and fiber enhance the thermal cooling rate of the fiber at higher temperature. Therefore, $830 \mathrm{~mW}$ and $498 \mathrm{~mW}$ laser power are needed to shift the resonance wavelength of the FBG the same amount $(2.0 \mathrm{~nm})$ at 6.3 and 749 Torr, respectively.

Typical thermal-based vacuum sensors have two operational modes: A constant resistance/temperature mode and a constant current/power mode. Figure 4 shows the FBG vacuum sensor operated in constant power mode. The input power of the argon laser was set at $145 \mathrm{~mW}$ and then $62 \mathrm{~mW}$. At 173 mTorr ambient pressure, these power levels produced resonant FBG wavelengths of 2.05 and $1.2 \mathrm{~nm}$, respectively. These wavelengths correspond to fiber temperatures of $179^{\circ} \mathrm{C}$ and $112{ }^{\circ} \mathrm{C}$. The resonant wavelength shift of the FBG as a function of the chamber pressure is shown in Fig. 4. As the chamber pressure increases, the resonant wavelength shift is reduced due to the increase of thermal exchange via convection between the gas molecules and the hot optical fiber. The FBG vacuum sensor exhibits two distinct operation regions. When the chamber pressure is below 1 Torr, the fiber resonance wavelength responds much more sensitively than that at higher pressures. When the pressure was increased from 173 to 1 Torr, the resonance wavelength

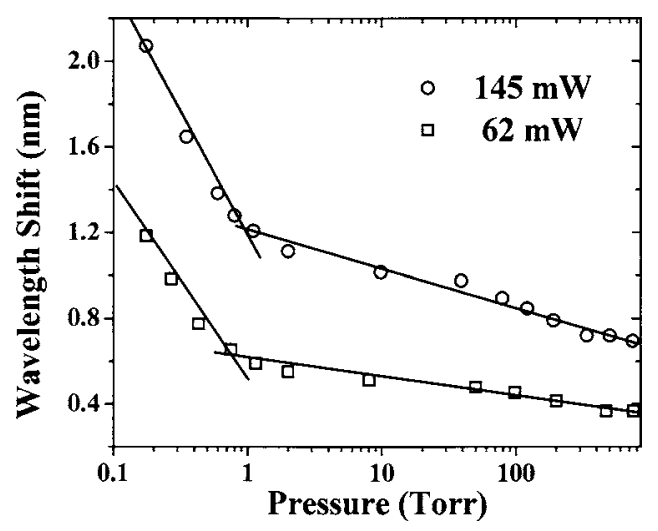

FIG 4. FBG vacuum sensor operation in constant power mode, the coupled laser powers are 62 and $145 \mathrm{~mW}$, respectively. 


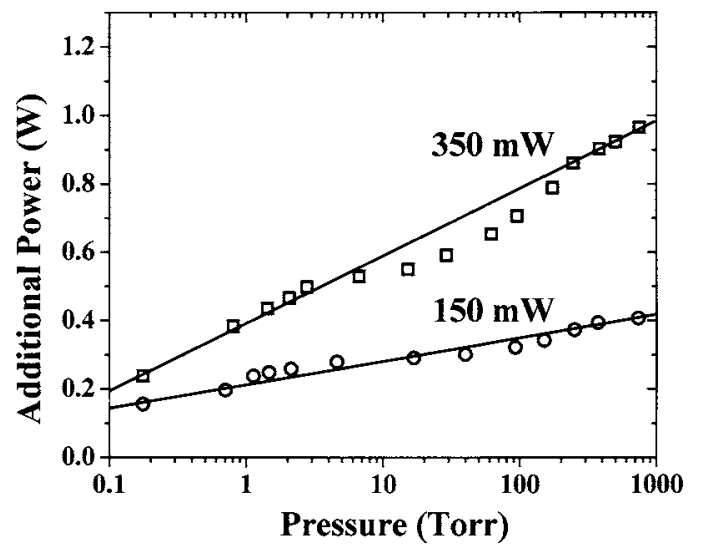

FIG. 5. FBG vacuum sensor operation in constant temperature mode while the FBG is preheated by the coupled laser power at 150 and $350 \mathrm{~mW}$, respectively.

of the FBG heated by $145 \mathrm{~mW}$ power was reduced by 0.85 $\mathrm{nm}$. In contrast, the resonance wavelength was only reduced by $0.44 \mathrm{~nm}$ for three orders of magnitude increase in pressure from 1 Torr to atmosphere. This is similar to the response of all thermally based vacuum sensors operating in the constant temperature mode. When the gas pressure is low $(<1$ Torr) so that the molecular mean free path is equal to or greater than the distance between the wall of the vacuum chamber and the hot fiber, the heat transfer is dictated by free molecular conduction, from the heated fiber to the ambient gas, followed by heat transfer between the gas and the cool wall of vacuum chamber. This process is proportional to the gas pressure. At higher pressures, heater transfer via convection between the heated fiber and ambient gas becomes significant. The heat transfer via convection is a nonlinear process and occurs at a much lower rate, which leads to reduced peak shifts. This problem can be solved by on-fiber packaging or using micro-structured fibers to eliminate thermal exchange by convection.

Figure 4 demonstrates the agility of the opticallypowered active sensor in tailoring its sensitivity with in-fiber power light. Given input laser power of either 145 or $62 \mathrm{~mW}$, the overall resonance wavelength shifts for pressures ranging from 173 mTorr to 1 Torr are 0.85 and $0.57 \mathrm{~nm}$, respectively. Thus, a $133 \%$ increase in laser power resulted in a $49 \%$ increase in the sensor's responsivity. While operating in the higher pressure regime from 1 Torr to atmosphere, the sensor's responsivity increase is more prominent. For the same magnitude power increase, the sensor responsivity increases by $100 \%$ from $0.22 \mathrm{~nm}$ shift for $62 \mathrm{nW}$ input power to 0.44 $\mathrm{nm}$ shift for $145 \mathrm{~mW}$ input power. Due to the limitations of the present vacuum system, we did not calibrate the FBG sensor at pressures below $170 \mathrm{~m}$ Torr. A traditional Pirani gauge with a similar thermal mass can operate at pressures down to $10^{-3}$ mTorr, and it is expected that the FBG vacuum sensor with $125 \mu \mathrm{m}$ diameter fiber can be operated effectively down to the same pressure.

The FBG vacuum sensor can also operate in a constant temperature mode. In this mode, the vacuum was calibrated by increasing the input laser power to maintain the temperature (and thus resonance wavelength) of the self-heated FBG. Calibration results are shown in Fig. 5 in which a $5 \mathrm{~mm}$ FBG was subsequently heated by 150 and $350 \mathrm{~mW}$ laser input power. In the constant temperature mode, The FBG sensor shows a single-exponential response to vacuum. This is in contrast to the FBG sensor operating in the constant temperature mode, in which two thermal response regimes were found. It is not clear why the sensor shows different behavior in the constant temperature modes. When the FBG sensor was heated by $350 \mathrm{~mW}$ laser power, nonlinear response was observed for the pressure from 3 to 200 Torr, probably also due to an increase in gas convection at higher pressures. The $120 \%$ increase in the initial laser power from 249 to $538 \mathrm{~mW}$ led to a $120 \%$ increase of the sensor responsivity.

The active fiber vacuum sensor demonstrated in this letter utilizes $125 \mu \mathrm{m}$ diameter double clad optical fiber. Unfortunately, the excess thermal mass of the fiber requires a large amount laser heating power $(50-500 \mathrm{~mW})$ and limits the response time of the sensor. The large distance between the hot fiber and instrument wall introduces convection heat transfer that reduces the linearity and sensitivity of the sensor at high pressures ( $>1$ Torr). These deficiencies can be improved upon by using a micro-structural fiber. ${ }^{8,9}$ In microstructure fibers, the fiber core is surrounded by several large air holes and supported by a thin "bridge" of material between the fiber core and the outer shell. This structure provides good thermal isolation between the fiber core and the outer shell. The optical power would be used to heat up the fiber core only. Vacuum is then measured by the thermal conduction between the hot fiber core and surrounding outer shell with less than $50 \mu \mathrm{m}$ air gap. This work will be presented elsewhere.

In conclusion, this work illustrates a solution to enhance the functionality of a passive fiber optical sensor component without sacrificing any of the intrinsic advantages of fiberbased devices. An optically heated fiber Bragg grating sensor was used to measure both temperature and pressure in a vacuum system. When the power laser is turned off, the grating is used as traditional temperature sensor. When the laser is turned on, the thermal response of optically heated grating was used to monitor ambient pressure. The sensitivity and dynamic range of optically powered fiber sensors can by actively adjusted by in-fiber light to gauge the vacuum pressure over four orders of magnitude. The double-clad fiber used in this work can deliver optical power to any point along the fiber. Using wavelength division multiplexing, a vacuum and temperature sensor array can be constructed in one fiber with tens of sensor elements using only one fiber feed-through.

${ }^{1}$ K. O. Hill, Y. Fujii, D. C. Johnson, and B. S. Kawasaki, Appl. Phys. Lett. 32, 647 (1978).

${ }^{2}$ A. Othonos, Rev. Sci. Instrum. 68, 4309 (1997).

${ }^{3}$ W. Liang, Y. Huang, Y. Xu, R. K. Lee, and A. Yariv, Appl. Phys. Lett. 86, $151122(2005)$

${ }^{4}$ K. P. Chen, L. Cashdollar, and W. Xu, Appl. Phys. Lett. 16, 1897 (2004).

${ }^{5}$ St. Wilfert and Chr. Edelmann, J. Vac. Sci. Technol. A 22, 309 (2004).

${ }^{6}$ S. N. Wang, K. Mizuno, M. Fujiyoshi, H. Funabashi, and J. Sakata, Appl. Phys. Lett. 19, 353 (2001).

${ }^{7}$ W. J. Alvesteffer, D. C. Jacobs, and D. H. Baker, J. Vac. Sci. Technol. A 13, 2980 (1995)

${ }^{8}$ P. Mach, M. Dolinski, K. W. Baldwin, J. A. Rogers, C. Kerbage, R. S. Windeler, and B. J. Eggleton, Appl. Phys. Lett. 80, 4294 (2002).

${ }^{9}$ C. Kerbage, P. Steinvurzel, P. Reyes, P. S. Westbrook, R. S. Windeler, A. Hale, and B. J. Eggleton, Opt. Lett. 27, 842 (2002). 